\title{
Dietary patterns and their associations with health behaviours in Korea
}

\author{
Eo Rin Cho ${ }^{1,2,3}$, Aesun Shin ${ }^{1, *}$, Sun-Young $\operatorname{Lim}^{1}$ and Jeongseon Kim ${ }^{1}$ \\ ${ }^{1}$ Cancer Epidemiology Branch, Division of Cancer Epidemiology and Management, Research Institute, \\ National Cancer Center, 323 Ilsan-ro, Ilsandong-gu, Goyang-si, Gyeonggi-do 410-769, Republic of Korea: \\ ${ }^{2}$ Department of Public Health, The Graduate School, Yonsei University, Republic of Korea: ${ }^{3}$ Institute of Human \\ Genomic Study, College of Medicine, Korea University, Seoul, Republic of Korea
}

Submitted 18 March 2010: Accepted 18 August 2010: First published online 19 0ctober 2010

\begin{abstract}
Objective: Dietary habits, including dietary patterns, have been associated with the risk of chronic diseases, including cancer. The objective of the present study was to evaluate Korean dietary patterns as assessed by using an FFQ and associations of dietary patterns with lifestyle risk factors.

Design: Dietary patterns were analysed by factor analysis using a sixteen-group FFQ. The associations between dietary patterns and lifestyle risk factors were investigated by logistic regression analysis.

Setting: The National Cancer Center in South Korea.

Subjects: The study population included 11440 participants aged $\geq 30$ years who were recruited between 2002 and 2007.

Results: Compared with the lowest quartile intake of each dietary pattern, current smoking was positively associated with the Western pattern $(\mathrm{OR}=1.55$ for the highest quartile, 95\% CI 1.27, 1.88; $P<0.001)$ and the traditional pattern $(\mathrm{OR}=$ $1 \cdot 34,95 \% \mathrm{CI} 1 \cdot 11,1 \cdot 62 ; P=0 \cdot 002)$ in men, but was inversely associated with the healthy pattern in both genders $(P<0.001)$ and the traditional pattern $(\mathrm{OR}=0.52$, $95 \%$ CI $0.36,0 \cdot 75 ; P=0.001)$ in women. Alcohol consumption was positively associated with all patterns in both genders, while no association was observed with the healthy pattern in women. Physical activity and dietary supplement use were positively associated with all patterns in both genders, with the exception of physical activity in women, which showed an inverse association with the traditional pattern.

Conclusions: Dietary patterns are strongly associated with health behaviours. The possible confounding effect of other risk behaviours should be appropriately considered when conducting nutritional epidemiological studies.
\end{abstract}

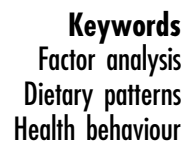

Although traditionally nutritional research has focused primarily on single nutrients or individual foods, interest is growing in dietary patterns that consider the complexity of the overall diet ${ }^{(1,2)}$. An exploratory approach using factor or cluster analysis empirically identifies patterns that represent actual eating behaviours of the study population. Typically, analyses extract two to six patterns that reflect different dietary compositions ${ }^{(3)}$.

Recently, several experimental, clinical and epidemiological studies have examined dietary patterns and found that patterns reflecting certain eating habits are associated with the risk of chronic diseases ${ }^{(2,4-7)}$, including cancer $^{(8,9)}$. Dietary habits are also closely related to other health-related behaviours. Current smoking is generally inversely related to a healthy diet ${ }^{(10-13)}$; alcohol consumption is positively related to diets with high intakes of fat and meat, but inversely related to a prudent diet ${ }^{(14-17)}$; and dietary supplement use is often associated with a healthy diet that reflects high intakes of skimmed milk, yoghurt, juice, cereals, rice, chicken, fruit and cod liver oil ${ }^{(11,18,19)}$. However, most studies of these factors have been conducted in North America or Europe, whereas only a few studies have investigated Asian populations ${ }^{(15,20)}$, and the results may not apply to the Korean population whose dietary habits are different from patterns in Western countries.

Thus, the purpose of the present study was to evaluate Korean dietary patterns by using an FFQ and to assess the association of dietary patterns with lifestyle risk factors, such as smoking, alcohol consumption, physical activity and dietary supplement use.

\section{Experimental methods}

\section{Study participants}

The source population comprised 14531 men and women who underwent cancer screening examinations at 
the Center for Cancer Prevention and Detection at the National Cancer Center in South Korea from August 2002 to May 2007. Participants who failed to complete information for all sixteen food groups in the FFQ or who were $<30$ years of age were excluded. After these exclusions, 11440 participants (6434 men and 5006 women) remained for the analyses. Written informed consent was obtained from all participants, and all procedures were approved by the Institutional Review Board of the National Cancer Center.

\section{Data collection}

All participants were asked to complete a self-administered questionnaire about their sociodemographic characteristics (e.g. age, education and household income), cigarette smoking habit, alcohol consumption habit, physical activity, dietary supplement use, personal medical history and medication use. The average alcohol consumption amount (g/d) was calculated by summing the beverage-specific amount consumed as reported on the FFQ, average consumption frequency and the volume of one standard drink for each type of alcoholic beverage (beer, hard liquor, wine and traditional drinks, including soju and Korean rice wine).

At the time of screening, for each participant, height and weight were measured using the InBody 3.0 (Biospace, Seoul, Korea) body composition analyser, and BMI was calculated as weight $(\mathrm{kg})$ divided by the square of height $\left(\mathrm{m}^{2}\right)$. Three categories were constructed for smoking habits (non-, ex- and current) and alcohol consumption (non-, light and heavy), and two categories each were constructed for participation in leisure-time physical activity, dietary supplement use and past medical history of hypertension and diabetes (yes or no).

\section{Dietary pattern derivation}

Dietary information was collected using an FFQ. The FFQ consisted of sixteen food groups: cereals, salted vegetables and seafood, light-coloured vegetables, green-yellow vegetables, seaweeds, fruits, grilled meat and seafood, healthy protein foods, dairy products, bonefish, fried foods, cholesterol-rich foods, animal fat-rich foods, sweet foods, fast foods and caffeinated drinks (see Appendix). All participants were asked to record their current intake frequency for each food group according to the following categories: consumed rarely, once monthly, 2-3 times/ month, once weekly, 2-3 times/week, 4-6 times/week, once daily or $>2$ times/d. In our previous study, the validity of this FFQ was compared to the $3 \mathrm{~d}$ dietary record method as a reference standard for 1401 participants from the source cohort. The cross-classification of the tertile categories by using these two methods showed good agreement (range: 38-96\%, depending on the food group). Generally, there was a trend that participants who reported a high frequency of intake on the brief FFQ also reported a high amount of intake of food in the $3 \mathrm{~d}$ dietary records. The highest agreement was observed for cereals (68.7\%), dairy foods $(59 \cdot 1 \%)$, fruits $(55 \cdot 6 \%)$ and caffeinated drinks $(61 \cdot 8 \%)^{(21)}$.

\section{Statistical analyses}

Dietary patterns were derived by using factor analysis, with the sixteen food groups entered into the analysis by the frequency of intake. The PROC FACTOR procedure in the SAS statistical software package version 9·1 (SAS Institute, Cary, NC, USA) was applied to perform the analysis. This procedure uses factor analysis and orthogonal rotation, using the Varimax option in SAS, to derive non-correlated factors and to render the results more easily interpretable. To determine which number of factors to retain, we examined both the scree plots and the factors themselves to see which set of factors most meaningfully described distinct food intake patterns. We considered components with an eigenvalue of $>1 \cdot 0$. This served to limit the number of factors, as well as to better identify the three most meaningful factors. Factor loadings were calculated for each food group across the three factors. Factors were thereby interpreted as dietary patterns and named after the food groups with a loading of $>0 \cdot 2$. A dietary factor score for each individual was then calculated by summing the consumption of food groups weighted by the factor loadings.

Dietary factor scores were categorized into quartiles separately for men and women based on the distribution of the study population, and linear regression analysis was performed to evaluate the association between dietary pattern categories and health behaviour variables with adjustments for age. $P$ for trend was calculated from generalized linear models with adjustments for age as a continuous variable and from Mantel-Haenszel $\chi^{2}$ tests with adjustments for age group as a categorical variable. To assess associations between dietary patterns and health behaviours, polytomous multiple logistic regression models were used to calculate the OR and 95\% CI for each quartile. $P$ for trend in the OR was calculated with the dietary pattern categories as a continuous variable. Analyses were conducted using the SAS software version $9 \cdot 1$ (SAS Institute). All analyses were performed separately for men and women, and a two-sided $P$ value of $<0.05$ was considered to be statistically significant.

\section{Results}

Among men and women aged $\geq 30$ years who were recruited between 2002 and 2007 from the National Cancer Center in South Korea, 11440 participants were included in the final analysis (6434 men and 5006 women). Table 1 shows the three main dietary patterns and the factorloading matrix between food groups. The larger the loading of a given food group to the factor, the greater the contribution of that food group to a specific factor. Dietary pattern 1 was characterized by high consumption of fast foods, animal fat-rich foods, fried foods, grilled 
Table 1 Factor-loading matrix for the major dietary patterns identified by factor analysis

\begin{tabular}{|c|c|c|c|c|c|c|}
\hline & \multicolumn{3}{|c|}{ Men $(n$ 6434) } & \multicolumn{3}{|c|}{ Women (n 5006) } \\
\hline & $\begin{array}{l}\text { Pattern } 1 \\
\text { (Western) }\end{array}$ & $\begin{array}{c}\text { Pattern } 2 \\
\text { (healthy) }\end{array}$ & $\begin{array}{l}\text { Pattern } 3 \\
\text { (traditional) }\end{array}$ & $\begin{array}{l}\text { Pattern } 1 \\
\text { (Western) }\end{array}$ & $\begin{array}{r}\text { Pattern } 2 \\
\text { (healthy) }\end{array}$ & $\begin{array}{c}\text { Pattern } 3 \\
\text { (traditional) }\end{array}$ \\
\hline Fast foods & $0 \cdot 70$ & & & $0 \cdot 72$ & & \\
\hline Animal fat-rich foods & 0.67 & & & $0 \cdot 71$ & & \\
\hline Fried foods & 0.66 & $0 \cdot 22$ & & $0 \cdot 61$ & $0 \cdot 21$ & \\
\hline Grilled meat and seafood & 0.59 & & & 0.54 & & \\
\hline Cholesterol-rich foods & 0.53 & 0.33 & & 0.51 & 0.33 & \\
\hline Sweet foods & 0.51 & & & 0.54 & & \\
\hline Caffeinated drinks & 0.27 & & & 0.35 & & \\
\hline Green-yellow vegetables & & $0 \cdot 64$ & 0.28 & & 0.58 & $0 \cdot 30$ \\
\hline Seaweeds & & 0.58 & & & 0.55 & 0.21 \\
\hline Healthy protein foods & & 0.56 & & & 0.58 & \\
\hline Bonefish & & 0.53 & & & 0.54 & \\
\hline Fruits & & 0.48 & & & 0.47 & \\
\hline Dairy products & & $0 \cdot 31$ & & & 0.34 & \\
\hline Salted vegetables and seafood & & & 0.63 & & & 0.66 \\
\hline Cereals & & & 0.58 & & & $0 \cdot 60$ \\
\hline Light-coloured vegetables & & 0.43 & 0.55 & & 0.38 & 0.56 \\
\hline
\end{tabular}

For the sake of simplicity, factor loadings of $<0.20$ are not listed.

meat and seafood (barbecue), cholesterol-rich foods, sweet foods and caffeinated drinks. We named this pattern the 'Western pattern'. Dietary pattern 2 was characterized by high consumption of green-yellow vegetables, seaweeds, healthy protein foods, bonefish, fruit and dairy products, and we named this pattern the 'healthy pattern'. Dietary pattern 3 was characterized by high consumption of salted vegetables and seafood, cereals and light-coloured vegetables, and we named this pattern the 'traditional pattern'. The major dietary patterns identified separately for men and women proved to be similar. Each of the three patterns explained $15 \cdot 3 \%, 13 \cdot 0 \%$ and $7 \cdot 8 \%$ of variance of food frequency consumption in men and $15 \cdot 3 \%, 12 \cdot 2 \%$ and $8.3 \%$ in women, respectively.

Demographic characteristics and lifestyle factors are presented as means (SD) or numbers and percentages stratified by quartiles of factor scores for each dietary pattern (Table 2). Among both men and women, participants with a higher healthy dietary pattern score tended to have a higher educational level and household income, to smoke less and to report more physical activity and dietary supplement use. Participants with a higher Western dietary pattern score were younger and more likely to smoke and drink, and men were more likely to have a higher BMI. Participants with a higher traditional dietary pattern score were more likely to have a higher educational level and household income and to be involved in regular leisuretime physical activity.

The results of multivariate logistic regression analyses of the association between dietary patterns and health behaviours such as smoking, alcohol consumption, regular exercise and dietary supplement use are presented in Table 3 for men and in Table 4 for women. Current smoking was positively associated with the Western dietary pattern $(P$ for trend $<0 \cdot 001)$ and the traditional dietary pattern ( $P$ for trend $=0 \cdot 002$ ) in men. However, current smoking was inversely associated with the healthy dietary pattern in both genders $(P$ for trend $<0 \cdot 001)$ and with the traditional dietary pattern ( $P$ for trend $=$ $0 \cdot 001)$ in women. Alcohol consumption was positively associated with all patterns in men, with the exception of light drinking, which was inversely associated with the Western dietary pattern ( $P$ for trend $=0 \cdot 044$ ). In women, heavy drinking was positively associated with the Western dietary pattern ( $P$ for trend $=0 \cdot 007$ ), and light drinking was positively associated with the traditional dietary pattern ( $P$ for trend $=0 \cdot 001$, while there was no association observed with the healthy pattern. Physical activity and dietary supplement use were positively associated with all patterns in both genders, with the exception of physical activity, which was inversely associated with the traditional dietary pattern in women.

\section{Discussion}

In the present study of Korean adults, we identified three major dietary patterns, Western, healthy and traditional, and we evaluated their relationship with health behaviours. The three dietary patterns identified in the present study were similar to the dietary patterns found by previous studies conducted among Asian and Western populations using factor analysis $(10,14,15,22)$.

The Western pattern and the healthy pattern (or 'prudent pattern') have been reported by many other studies. The main contributors to the Western dietary pattern are typically meats, fats, fast food, sweets, grains, butter, eggs, potatoes and sugar-containing foods ${ }^{(8,23,24)}$. The main contributors to the healthy pattern are vegetables, fruit, fish and poultry $^{(9,12,23-26)}$. Several studies have also reported a traditional 
Table 2 Characteristics of study participants according to quartiles of factor scores for each pattern

\begin{tabular}{|c|c|c|c|c|c|c|c|c|c|c|}
\hline & \multicolumn{4}{|c|}{ Men } & \multirow[b]{3}{*}{$P$ for trend ${ }^{*}$} & \multicolumn{4}{|c|}{ Women } & \multirow[b]{3}{*}{$P$ for trend ${ }^{*}$} \\
\hline & \multicolumn{2}{|c|}{$\begin{array}{l}\text { Quartile } 1 \\
(n \text { 1608) }\end{array}$} & \multicolumn{2}{|c|}{$\begin{array}{c}\text { Quartile } 4 \\
(n 1609)\end{array}$} & & \multicolumn{2}{|c|}{$\begin{array}{l}\text { Quartile } 1 \\
(n 1251)\end{array}$} & \multicolumn{2}{|c|}{$\begin{array}{l}\text { Quartile } 4 \\
(n \text { 1252) }\end{array}$} & \\
\hline & $n$ & $\%$ & $n$ & $\%$ & & $n$ & $\%$ & $n$ & $\%$ & \\
\hline \multicolumn{11}{|l|}{ Western pattern } \\
\hline Age (years) $\dagger$ & $53 \cdot 12$ & $9 \cdot 30$ & $44 \cdot 52$ & $9 \cdot 20$ & $<0.001$ & $53 \cdot 06$ & $8 \cdot 55$ & $43 \cdot 08$ & $8 \cdot 56$ & $<0.001$ \\
\hline BMI $\left(\mathrm{kg} / \mathrm{m}^{2}\right)+$ & $24 \cdot 38$ & $2 \cdot 58$ & $24 \cdot 86$ & $2 \cdot 94$ & $<0.001$ & $23 \cdot 55$ & $3 \cdot 00$ & $22 \cdot 50$ & $2 \cdot 92$ & 0.916 \\
\hline \multicolumn{11}{|l|}{ Education level } \\
\hline Middle or less & 240 & $15 \cdot 09$ & 78 & $4 \cdot 87$ & $<0.001$ & 414 & 33.91 & 107 & $8 \cdot 60$ & $<0.001$ \\
\hline High school & 524 & $32 \cdot 96$ & 375 & $23 \cdot 39$ & & 502 & $41 \cdot 11$ & 420 & $33 \cdot 76$ & \\
\hline College or more & 826 & 51.95 & 1150 & $71 \cdot 74$ & & 305 & 24.98 & 717 & $57 \cdot 64$ & \\
\hline Household incomeł & & & & & & & & & & \\
\hline$<2000$ & 285 & $20 \cdot 26$ & 110 & $7 \cdot 71$ & $<0.001$ & 298 & $30 \cdot 19$ & 130 & $12 \cdot 25$ & $<0.001$ \\
\hline 2000-3999 & 481 & $34 \cdot 19$ & 491 & $34 \cdot 43$ & & 340 & $34 \cdot 45$ & 343 & $32 \cdot 33$ & \\
\hline$>4000$ & 641 & $45 \cdot 56$ & 825 & $57 \cdot 85$ & & 349 & $35 \cdot 36$ & 588 & $55 \cdot 42$ & \\
\hline Smoking & & & & & & & & & & \\
\hline Non-smokers & 407 & $25 \cdot 78$ & 279 & $17 \cdot 49$ & $<0.001$ & 946 & $92 \cdot 93$ & 982 & $88 \cdot 31$ & 0.001 \\
\hline Ex-smokers & 584 & 36.99 & 445 & $27 \cdot 90$ & & 20 & 1.96 & 43 & $3 \cdot 87$ & \\
\hline Current smokers & 588 & $37 \cdot 24$ & 871 & $54 \cdot 61$ & & 52 & $5 \cdot 11$ & 87 & $7 \cdot 82$ & \\
\hline $\begin{array}{l}\text { Alcohol consumption } \\
\text { Non-drinkers }(0 \mathrm{~g} / \mathrm{d})\end{array}$ & 197 & $17 \cdot 30$ & 153 & $12 \cdot 91$ & $<0.001$ & 658 & $66 \cdot 53$ & 487 & 48.99 & $<0.001$ \\
\hline Light drinkers $(<12 \mathrm{~g} / \mathrm{d})$ & 401 & $35 \cdot 21$ & 289 & $24 \cdot 39$ & & 291 & $29 \cdot 42$ & 415 & $41 \cdot 75$ & \\
\hline Heavy drinkers $(\geq 12 \mathrm{~g} / \mathrm{d})$ & 541 & $47 \cdot 50$ & 743 & $62 \cdot 70$ & & 40 & $4 \cdot 04$ & 92 & $9 \cdot 26$ & \\
\hline Physical activity & & & & & & & & & & \\
\hline No & 546 & $38 \cdot 78$ & 461 & $30 \cdot 63$ & $<0.001$ & 538 & $52 \cdot 85$ & 577 & $51 \cdot 52$ & 0.742 \\
\hline Yes & 862 & $61 \cdot 22$ & 1044 & $69 \cdot 37$ & & 480 & $47 \cdot 15$ & 543 & $48 \cdot 48$ & \\
\hline History of hypertension§ & & & & & & & & & & \\
\hline No & 1241 & $77 \cdot 18$ & 1404 & $87 \cdot 26$ & $<0.001$ & 1024 & $81 \cdot 85$ & 1166 & $93 \cdot 13$ & $<0.001$ \\
\hline Yes & 367 & $22 \cdot 82$ & 205 & $12 \cdot 74$ & & 227 & $18 \cdot 15$ & 86 & $6 \cdot 87$ & \\
\hline History of diabetesł & & & & & & & & & & \\
\hline No & 1439 & $89 \cdot 49$ & 1517 & $94 \cdot 28$ & $<0.001$ & 1166 & $93 \cdot 21$ & 1231 & $98 \cdot 32$ & $<0.001$ \\
\hline Yes & 169 & $10 \cdot 51$ & 92 & $5 \cdot 72$ & & 85 & $6 \cdot 79$ & 21 & $1 \cdot 68$ & \\
\hline Dietary supplement use & & & & & & & & & & \\
\hline No & 1099 & $68 \cdot 35$ & 1095 & $68 \cdot 06$ & $0 \cdot 708$ & 701 & $56 \cdot 04$ & 715 & $57 \cdot 11$ & $0 \cdot 347$ \\
\hline Yes & 509 & $31 \cdot 65$ & 514 & 31.95 & & 55 & $43 \cdot 96$ & 537 & $42 \cdot 89$ & \\
\hline Healthy pattern & & & & & & & & & & \\
\hline Age (years) $\dagger$ & $47 \cdot 15$ & $9 \cdot 87$ & $50 \cdot 22$ & $9 \cdot 64$ & $<0.001$ & $47 \cdot 01$ & $9 \cdot 65$ & $49 \cdot 37$ & $9 \cdot 27$ & $<0.001$ \\
\hline $\mathrm{BMI}\left(\mathrm{kg} / \mathrm{m}^{2}\right) \dagger$ & 24.49 & $2 \cdot 77$ & $24 \cdot 57$ & $2 \cdot 67$ & 0.229 & 23.08 & $3 \cdot 11$ & 23.03 & $2 \cdot 86$ & 0.009 \\
\hline Education level & & & & & & & & & & \\
\hline Middle or less & 217 & $13 \cdot 62$ & 112 & $7 \cdot 02$ & $<0.001$ & 362 & $29 \cdot 43$ & 186 & $15 \cdot 11$ & $<0.001$ \\
\hline High school & 516 & $32 \cdot 39$ & 364 & $22 \cdot 81$ & & 506 & $41 \cdot 14$ & 471 & $38 \cdot 26$ & \\
\hline College or more & 860 & 53.99 & 1120 & $70 \cdot 18$ & & 362 & $29 \cdot 43$ & 574 & $46 \cdot 63$ & \\
\hline Household income & & & & & & & & & & \\
\hline$<2000$ & 288 & $19 \cdot 81$ & 113 & $8 \cdot 11$ & $<0.001$ & 271 & $26 \cdot 06$ & 134 & $13 \cdot 35$ & $<0.001$ \\
\hline 2000-3999 & 551 & $37 \cdot 90$ & 429 & $30 \cdot 77$ & & 353 & 33.94 & 349 & $34 \cdot 76$ & \\
\hline$>4000$ & 615 & $42 \cdot 30$ & 852 & $61 \cdot 12$ & & 416 & $40 \cdot 00$ & 521 & $51 \cdot 89$ & \\
\hline Smoking & & & & & & & & & & \\
\hline Non-smokers & 284 & $17 \cdot 94$ & 380 & $24 \cdot 11$ & $<0.001$ & 950 & $87 \cdot 96$ & 983 & $93 \cdot 26$ & $<0.001$ \\
\hline Ex-smokers & 488 & $30 \cdot 83$ & 564 & $35 \cdot 79$ & & 36 & 3.33 & 24 & $2 \cdot 28$ & \\
\hline Current smokers & 811 & $51 \cdot 23$ & 632 & $40 \cdot 10$ & & 94 & $8 \cdot 70$ & 47 & $4 \cdot 46$ & \\
\hline Alcohol consumption & & & & & & & & & & \\
\hline Non-drinkers $(0 \mathrm{~g} / \mathrm{d})$ & 177 & $16 \cdot 48$ & 156 & $12 \cdot 77$ & $0 \cdot 151$ & 492 & $53 \cdot 36$ & 613 & $58 \cdot 55$ & 0.016 \\
\hline Light drinkers $(<12 \mathrm{~g} / \mathrm{d})$ & 309 & $28 \cdot 77$ & 381 & $31 \cdot 18$ & & 354 & $38 \cdot 39$ & 366 & 34.96 & \\
\hline Heavy drinkers $(\geq 12 \mathrm{~g} / \mathrm{d})$ & 588 & $54 \cdot 75$ & 685 & $56 \cdot 06$ & & 76 & $8 \cdot 24$ & 68 & $6 \cdot 49$ & \\
\hline Physical activity & & & & & & & & & & \\
\hline No & 662 & $45 \cdot 88$ & 411 & $27 \cdot 92$ & $<0.001$ & 651 & $61 \cdot 71$ & 465 & $43 \cdot 70$ & $<0.001$ \\
\hline Yes & 781 & $54 \cdot 12$ & 1061 & $72 \cdot 08$ & & 404 & $38 \cdot 29$ & 599 & $56 \cdot 30$ & \\
\hline History of hypertension§ & & & & & & & & & & \\
\hline No & 1363 & $84 \cdot 76$ & 1297 & $80 \cdot 66$ & $<0.001$ & 1093 & $87 \cdot 37$ & 1086 & $86 \cdot 81$ & 0.612 \\
\hline Yes & 245 & $15 \cdot 24$ & 311 & $19 \cdot 34$ & & 158 & $12 \cdot 63$ & 165 & $13 \cdot 19$ & \\
\hline History of diabetesł & & & & & & & & & & \\
\hline No & 1514 & $94 \cdot 15$ & 1459 & $90 \cdot 73$ & $<0.001$ & 1205 & $96 \cdot 32$ & 1197 & $95 \cdot 68$ & 0.382 \\
\hline Yes & 94 & $5 \cdot 85$ & 149 & $9 \cdot 27$ & & 46 & $3 \cdot 68$ & 54 & $4 \cdot 32$ & \\
\hline Dietary supplement use & & & & & & & & & & \\
\hline No & 1230 & $76 \cdot 49$ & 1001 & $62 \cdot 25$ & $<0.001$ & 804 & $64 \cdot 27$ & 606 & $48 \cdot 44$ & $<0.001$ \\
\hline Yes & 378 & $23 \cdot 51$ & 607 & $37 \cdot 75$ & & 447 & $35 \cdot 73$ & 645 & $51 \cdot 56$ & \\
\hline Traditional pattern & & & & & & & & & & \\
\hline Age (years) $†$ & $49 \cdot 09$ & $9 \cdot 92$ & $48 \cdot 19$ & $9 \cdot 58$ & 0.017 & $48 \cdot 27$ & $9 \cdot 13$ & $47 \cdot 93$ & $9 \cdot 41$ & $0 \cdot 288$ \\
\hline BMI $\left(\mathrm{kg} / \mathrm{m}^{2}\right) \dagger$ & $24 \cdot 51$ & $2 \cdot 80$ & $24 \cdot 45$ & $2 \cdot 79$ & $0 \cdot 169$ & $23 \cdot 15$ & $2 \cdot 86$ & $23 \cdot 11$ & $3 \cdot 20$ & $0 \cdot 797$ \\
\hline
\end{tabular}




\begin{tabular}{|c|c|c|c|c|c|c|c|c|c|c|}
\hline & \multicolumn{4}{|c|}{ Men } & \multirow[b]{3}{*}{$P$ for trend ${ }^{*}$} & \multicolumn{4}{|c|}{ Women } & \multirow[b]{3}{*}{$P$ for trend } \\
\hline & \multicolumn{2}{|c|}{$\begin{array}{l}\text { Quartile } 1 \\
(n 1608)\end{array}$} & \multicolumn{2}{|c|}{$\begin{array}{c}\text { Quartile } 4 \\
(n 1609)\end{array}$} & & \multicolumn{2}{|c|}{$\begin{array}{c}\text { Quartile 1 } \\
\text { (n 1251) }\end{array}$} & \multicolumn{2}{|c|}{$\begin{array}{c}\text { Quartile } 4 \\
(n 1252)\end{array}$} & \\
\hline & $n$ & $\%$ & $n$ & $\%$ & & $n$ & $\%$ & $n$ & $\%$ & \\
\hline \multicolumn{11}{|l|}{ Education level } \\
\hline Middle or less & 191 & $12 \cdot 00$ & 138 & $8 \cdot 64$ & \multirow[t]{3}{*}{$<0.001$} & 324 & $26 \cdot 32$ & 246 & $20 \cdot 05$ & \multirow[t]{3}{*}{$<0.001$} \\
\hline High school & 514 & $32 \cdot 29$ & 427 & $26 \cdot 74$ & & 522 & $42 \cdot 40$ & 480 & $39 \cdot 12$ & \\
\hline College or more & 887 & $55 \cdot 72$ & 1032 & $64 \cdot 62$ & & 385 & $31 \cdot 28$ & 501 & $40 \cdot 83$ & \\
\hline \multicolumn{11}{|l|}{ Household incomeł } \\
\hline$<2000$ & 245 & $16 \cdot 98$ & 168 & $12 \cdot 03$ & \multirow[t]{3}{*}{$<0.001$} & 230 & $22 \cdot 55$ & 176 & $17 \cdot 14$ & \multirow[t]{3}{*}{$<0.001$} \\
\hline 2000-3999 & 508 & $35 \cdot 20$ & 484 & 34.65 & & 380 & $37 \cdot 25$ & 332 & $32 \cdot 33$ & \\
\hline$>4000$ & 690 & $47 \cdot 82$ & 745 & $53 \cdot 33$ & & 410 & $40 \cdot 20$ & 519 & $50 \cdot 54$ & \\
\hline \multicolumn{11}{|l|}{ Smoking } \\
\hline Non-smokers & 369 & $23 \cdot 34$ & 287 & $18 \cdot 04$ & \multirow{3}{*}{0.001} & 911 & $89 \cdot 40$ & 1023 & $92 \cdot 92$ & \multirow{3}{*}{0.001} \\
\hline Ex-smokers & 474 & $29 \cdot 98$ & 499 & $31 \cdot 36$ & & 28 & $2 \cdot 75$ & 31 & $2 \cdot 82$ & \\
\hline Current smokers & 738 & $46 \cdot 68$ & 805 & $50 \cdot 60$ & & 80 & $7 \cdot 85$ & 47 & $4 \cdot 27$ & \\
\hline \multicolumn{11}{|l|}{ Alcohol consumption } \\
\hline Non-drinkers $(0 \mathrm{~g} / \mathrm{d})$ & 182 & $18 \cdot 35$ & 159 & $12 \cdot 67$ & \multirow[t]{3}{*}{$<0.001$} & 555 & $60 \cdot 52$ & 572 & $55 \cdot 21$ & \multirow[t]{3}{*}{0.763} \\
\hline Light drinkers $(<12 \mathrm{~g} / \mathrm{d})$ & 299 & $30 \cdot 14$ & 380 & $30 \cdot 28$ & & 284 & $30 \cdot 97$ & 407 & $39 \cdot 29$ & \\
\hline Heavy drinkers $(\geq 12 \mathrm{~g} / \mathrm{d})$ & 511 & $51 \cdot 51$ & 716 & $57 \cdot 05$ & & 78 & $8 \cdot 51$ & 57 & $5 \cdot 50$ & \\
\hline \multicolumn{11}{|l|}{ Physical activity } \\
\hline No & 557 & $39 \cdot 12$ & 523 & $35 \cdot 51$ & \multirow[t]{2}{*}{0.011} & 517 & $49 \cdot 52$ & 588 & 53.94 & \multirow[t]{2}{*}{0.038} \\
\hline Yes & 867 & $60 \cdot 88$ & 950 & $64 \cdot 49$ & & 527 & $50 \cdot 48$ & 502 & $46 \cdot 06$ & \\
\hline \multicolumn{11}{|l|}{ History of hypertension§ } \\
\hline No & 1338 & $83 \cdot 16$ & 1342 & $83 \cdot 46$ & \multirow[t]{2}{*}{0.506} & 1105 & $88 \cdot 26$ & 1096 & $87 \cdot 54$ & \multirow[t]{2}{*}{0.612} \\
\hline Yes & 271 & $16 \cdot 84$ & 266 & $16 \cdot 54$ & & 147 & $11 \cdot 74$ & 156 & $12 \cdot 46$ & \\
\hline \multicolumn{11}{|l|}{ History of diabetesł } \\
\hline No & 1494 & $92 \cdot 85$ & 1498 & $93 \cdot 16$ & \multirow[t]{2}{*}{0.411} & 1210 & $96 \cdot 65$ & 1200 & $95 \cdot 85$ & $0 \cdot 278$ \\
\hline Yes & 115 & $7 \cdot 15$ & 110 & $6 \cdot 84$ & & 42 & 3.35 & 52 & $4 \cdot 15$ & \\
\hline Dietary supplement use & & & & & & & & & & \\
\hline No & 1189 & $73 \cdot 90$ & 1061 & $65 \cdot 98$ & $<0.001$ & 749 & $59 \cdot 82$ & 709 & $56 \cdot 63$ & 0.081 \\
\hline Yes & 420 & $26 \cdot 10$ & 547 & $34 \cdot 02$ & & 503 & $40 \cdot 18$ & 543 & $43 \cdot 37$ & \\
\hline
\end{tabular}

${ }^{\star} P$ for trend was calculated from a generalized linear model with adjustments for age for continuous variables, and a Mantel-Haenszel $\chi^{2}$ test with adjustments for the age group for categorical variables.

tAge and BMI data are mean and standard deviation.

fUnit is thousand won (month.

SHypertension was defined as the use of antihypertensive medication or a history of hypertension. Diabetes was defined as the use of any diabetes medication or a history of diabetes.

dietary pattern ${ }^{(12,15,20,22,27,28)}$. In a study of 1441 Korean children, in which thirty-three food groups were created and entered into a factor analysis, the traditional (Korean) pattern included vegetables, seaweeds, beans, fruits, milk and dairy products $^{(20)}$. A study of the dietary pattern of 637 Korean Americans found that the traditional Korean pattern was characterized by high intake of traditional Korean dishes, such as soyabean paste stew, anchovies, Korean-style grains, tofu, vegetables, kimchi, salted/fermented fish, seaweeds, Korean-style soups, red meat, other seafood (shrimp, squid, clams, oyster, etc) and fish ${ }^{(22)}$. In Japan, the traditional pattern includes pickled vegetables, salted fish and roe, fish, rice and miso soup ${ }^{(15)}$. In contrast, in the Netherlands, the traditional pattern is characterized by higher consumption of red meat, potatoes, highly saturated added fats, coffee and beer and lower consumption of soya products, low-fat dairy, breakfast cereals, tea and fruits ${ }^{(27)}$. A traditional Norwegian dietary pattern consists mainly of two or three similar cold meals, usually open sandwiches, and one hot meal with either fish or meat served with potatoes and vegetables ${ }^{(28)}$. The traditional dietary pattern in the present study included salted foods, such as pickled vegetables, kimchi and rice.
Studies of dietary patterns conducted in other populations have noted the importance of evaluating associations of dietary patterns with health behaviours, such as smoking, alcohol consumption, physical activity and dietary supplement use. For smoking, our study found that current smoking was strongly associated with the traditional and Western dietary patterns in men, but was inversely associated with the healthy pattern in both genders. In general, previously conducted studies have found that current smoking is inversely related to a healthy dietary pattern ${ }^{(11,12)}$, whereas former smokers tend to have a healthier dietary pattern ${ }^{(29)}$. For example, in a population-based Dutch study of 4244 men, men who smoked $>20$ cigarettes/d had significantly lower intake of $\beta$-carotene and especially ascorbic acid compared with men who never smoked, which was attributed to an almost $60 \%$ lower fruit intake among smokers ${ }^{(30)}$. The European Prospective Investigation into Cancer and Nutrition (EPIC)-Potsdam study found that the 'fruit and vegetable' dietary pattern was negatively associated with smoking $(\beta=-0.188$ in men and -0.225 in women $)^{(10)}$. The Monitoring of Trends and Determinants 
Table 3 Dietary patterns according to lifestyle risk factors (smoking, alcohol consumption, physical activity and dietary supplement use status), by polytomous logistic regression analysis, in men

\begin{tabular}{|c|c|c|c|c|c|c|c|c|c|}
\hline & \multicolumn{2}{|c|}{ Quartile 1} & \multicolumn{2}{|c|}{ Quartile 2} & \multicolumn{2}{|c|}{ Quartile 3} & \multicolumn{2}{|c|}{ Quartile 4} & \multirow[b]{2}{*}{$P$ for trend } \\
\hline & OR & $95 \% \mathrm{Cl}$ & OR & $95 \% \mathrm{Cl}$ & OR & $95 \% \mathrm{Cl}$ & OR & $95 \% \mathrm{Cl}$ & \\
\hline \multicolumn{10}{|l|}{ Western pattern } \\
\hline \multicolumn{10}{|l|}{$\begin{array}{l}\text { Smoking } \\
\text { Non-smokers }\end{array}$} \\
\hline Ex-smokers & 1 & Ref. & $1 \cdot 27$ & $1 \cdot 05,1 \cdot 54$ & $1 \cdot 13$ & $0.93,1.37$ & $1 \cdot 22$ & $0.99,1.49$ & $0 \cdot 127$ \\
\hline Current smokers & 1 & Ref. & $1 \cdot 46$ & $1 \cdot 21,1 \cdot 76$ & $1 \cdot 22$ & $1 \cdot 01,1 \cdot 47$ & 1.55 & $1 \cdot 27,1 \cdot 88$ & $<0.001$ \\
\hline \multicolumn{10}{|l|}{ Alcohol consumptiont } \\
\hline Light drinkers & 1 & Ref. & $1 \cdot 12$ & $0.86,1 \cdot 45$ & 0.94 & $0.72,1 \cdot 23$ & 0.77 & $0.58,1.02$ & 0.044 \\
\hline Heavy drinkers & 1 & Ref. & $1 \cdot 27$ & $1 \cdot 00,1 \cdot 63$ & $1 \cdot 34$ & $1 \cdot 05,1 \cdot 73$ & $1 \cdot 28$ & $1 \cdot 00,1 \cdot 65$ & 0.053 \\
\hline \multicolumn{10}{|l|}{ Physical activity } \\
\hline $\begin{array}{l}\text { No } \\
\text { Yes }\end{array}$ & & & & & & & & & \\
\hline $\begin{array}{l}\text { Yes } \\
\text { Dietary supplement }\end{array}$ & 1 & Ref. & $1 \cdot 16$ & $1 \cdot 00,1 \cdot 35$ & $1 \cdot 39$ & $1 \cdot 19,1 \cdot 63$ & $1 \cdot 61$ & $1 \cdot 37,1 \cdot 90$ & $<0.001$ \\
\hline \multicolumn{10}{|c|}{ Dietary supplement use } \\
\hline Yes & 1 & Ref. & $1 \cdot 11$ & $0.95,1.29$ & $1 \cdot 26$ & $1 \cdot 08,1 \cdot 47$ & $1 \cdot 30$ & $1 \cdot 11,1 \cdot 52$ & $<0.001$ \\
\hline \multicolumn{10}{|l|}{ Healthy pattern } \\
\hline \multicolumn{10}{|l|}{$\begin{array}{l}\text { Smoking } \\
\text { Non-smoke }\end{array}$} \\
\hline Ex-smokers & 1 & Ref. & 0.89 & $0 \cdot 73,1 \cdot 10$ & 0.88 & $0.72,1.08$ & 0.84 & $0.69,1.02$ & 0.098 \\
\hline Current smokers & 1 & Ref. & 0.84 & $0.69,1.01$ & 0.85 & $0.70,1.03$ & 0.66 & $0.54,0.79$ & $<0.001$ \\
\hline \multicolumn{10}{|l|}{ Alcohol consumptiont } \\
\hline Non-drinkers & & & & & & & & & \\
\hline Light drinkers & 1 & Ref. & $1 \cdot 34$ & $1 \cdot 02,1 \cdot 74$ & $1 \cdot 25$ & $0.96,1.63$ & $1 \cdot 49$ & $1 \cdot 14,1 \cdot 94$ & 0.009 \\
\hline Heavy drinkers & 1 & Ref. & $1 \cdot 36$ & $1 \cdot 06,1 \cdot 74$ & $1 \cdot 35$ & $1 \cdot 06,1 \cdot 72$ & $1 \cdot 58$ & $1 \cdot 24,2 \cdot 04$ & 0.001 \\
\hline \multicolumn{10}{|l|}{ Physical activity } \\
\hline \multicolumn{10}{|l|}{ No } \\
\hline Yes & 1 & Ref. & $1 \cdot 50$ & $1 \cdot 29,1 \cdot 74$ & $2 \cdot 05$ & $1 \cdot 76,2 \cdot 39$ & $2 \cdot 16$ & $1 \cdot 86,2 \cdot 53$ & $<0.001$ \\
\hline \multirow{2}{*}{\multicolumn{10}{|c|}{$\begin{array}{l}\text { Dietary supplement use } \\
\text { No }\end{array}$}} \\
\hline & & & & & & & & & \\
\hline Yes & 1 & Ref. & 1.53 & $1 \cdot 31,1 \cdot 79$ & $1 \cdot 66$ & $1 \cdot 42,1 \cdot 94$ & $1 \cdot 85$ & $1 \cdot 59,2 \cdot 16$ & $<0.001$ \\
\hline \multicolumn{10}{|l|}{ Traditional pattern } \\
\hline \multicolumn{10}{|l|}{ Smoking } \\
\hline \multicolumn{10}{|l|}{ Non-smokers } \\
\hline Ex-smokers & 1 & Ref. & $1 \cdot 26$ & $1 \cdot 04,1 \cdot 53$ & $1 \cdot 30$ & $1 \cdot 07,1 \cdot 58$ & $1 \cdot 36$ & $1 \cdot 12,1 \cdot 66$ & 0.003 \\
\hline Current smokers & 1 & Ref. & 0.97 & $0 \cdot 81,1 \cdot 17$ & 1.02 & $0 \cdot 85,1 \cdot 22$ & $1 \cdot 34$ & $1 \cdot 11,1 \cdot 62$ & 0.002 \\
\hline \multirow{2}{*}{\multicolumn{10}{|c|}{$\begin{array}{l}\text { Alcohol consumptiont } \\
\text { Non-drinkers }\end{array}$}} \\
\hline Non-drinkers & & & & & & & & & \\
\hline Light drinkers & 1 & Ref. & $1 \cdot 43$ & $1 \cdot 10,1 \cdot 86$ & $1 \cdot 33$ & $1 \cdot 02,1 \cdot 73$ & $1 \cdot 44$ & $1 \cdot 11,1 \cdot 87$ & 0.028 \\
\hline Heavy drinkers & 1 & Ref. & $1 \cdot 43$ & $1 \cdot 12,1 \cdot 83$ & $1 \cdot 64$ & $1 \cdot 28,2 \cdot 09$ & $1 \cdot 54$ & $1 \cdot 21,1 \cdot 97$ & $<0.001$ \\
\hline \multicolumn{10}{|l|}{ Physical activity } \\
\hline No & & & & & & & & & \\
\hline Yes & 1 & Ref. & $1 \cdot 26$ & $1 \cdot 08,1 \cdot 46$ & $1 \cdot 48$ & $1 \cdot 27,1 \cdot 72$ & $1 \cdot 17$ & $1 \cdot 01,1 \cdot 37$ & 0.009 \\
\hline \multicolumn{10}{|c|}{ Dietary supplement use } \\
\hline $\begin{array}{l}\text { No } \\
\text { Yes }\end{array}$ & 1 & Rof & 1.55 & 1.331 .81 & 1.13 & 1.231 .67 & 1.50 & 1.201 .75 & - 0 P \\
\hline res & 1 & nel. & 1.55 & $1.33,1.81$ & $1 \cdot 43$ & & 1.50 & $1.29,1.15$ & $<0.001$ \\
\hline
\end{tabular}

Ref., reference category.

${ }^{*}$ Adjusted for age.

tNon-drinkers $(0 \mathrm{~g} / \mathrm{d})$; light drinkers $(<12 \mathrm{~g} / \mathrm{d})$; and heavy drinkers $(\geq 12 \mathrm{~g} / \mathrm{d})$.

in Cardiovascular Disease (MONICA) population survey of 976 men aged 45-64 years also found a negative association of smoking status $(P<0 \cdot 001)$ with a healthy dietary pattern ${ }^{(31)}$. Finally, the Multiethnic Cohort Study of 195298 participants residing in Hawaii and Los Angeles reported that current smokers showed a positive association with the fat and meat pattern (OR $=1 \cdot 67,95 \% \mathrm{CI}$ $1.62,1.72)$ and inverse associations with the vegetable $(\mathrm{OR}=0.66,95 \% \mathrm{CI} 0 \cdot 64,0 \cdot 68)$ and fruit and milk patterns $(\mathrm{OR}=0 \cdot 53,95 \% \text { CI } 0 \cdot 52,0 \cdot 55)^{(14)}$. Consistent with these findings, another study has shown that smokers are less likely than non-smokers to consume vegetables and milk or dairy foods. Smokers may consume more caffeinated or alcoholic beverages and meat products in order to enhance the taste of smoking ${ }^{(32)}$.

For alcohol consumption, we observed that heavy alcohol consumption was positively associated with the Western pattern in both genders, whereas light alcohol consumption was inversely associated with the Western pattern in men. Among 15073 university graduates enrolled in the Seguimiento Universidad de Navarra study in Spain, higher adherence to the Western dietary pattern was less likely to decrease participants' alcohol consumption during follow-up, whereas participants with 
Table 4 Dietary patterns according to lifestyle risk factors (smoking, alcohol consumption, physical activity, dietary supplement use status), by polytomous logistic regression analysis, in women

\begin{tabular}{|c|c|c|c|c|c|c|c|c|c|}
\hline & \multicolumn{2}{|c|}{ Quartile 1} & \multicolumn{2}{|c|}{ Quartile 2} & \multicolumn{2}{|c|}{ Quartile 3} & \multicolumn{2}{|c|}{ Quartile 4} & \multirow[b]{2}{*}{$P$ for trend ${ }^{*}$} \\
\hline & OR & $95 \% \mathrm{Cl}$ & OR & $95 \% \mathrm{Cl}$ & OR & $95 \% \mathrm{Cl}$ & OR & $95 \% \mathrm{Cl}$ & \\
\hline \multicolumn{10}{|l|}{ Western pattern } \\
\hline \multicolumn{10}{|l|}{ Smoking } \\
\hline \multicolumn{10}{|l|}{ Non-smokers } \\
\hline Ex-smokers & 1 & Ref. & $1 \cdot 42$ & $0 \cdot 80,2 \cdot 53$ & $1 \cdot 06$ & $0.57,1.96$ & $1 \cdot 70$ & $0.95,3.05$ & $0 \cdot 136$ \\
\hline $\begin{array}{l}\text { Current smokers } \\
\text { Alcohol consumption }\end{array}$ & 1 & Ref. & $0 \cdot 84$ & $0 \cdot 57,1 \cdot 25$ & 0.78 & $0 \cdot 53,1 \cdot 16$ & $0 \cdot 94$ & $0 \cdot 64,1 \cdot 38$ & $0 \cdot 854$ \\
\hline \multicolumn{10}{|l|}{ Non-drinkers } \\
\hline Light drinkers & 1 & Ref. & 0.97 & $0 \cdot 80,1 \cdot 19$ & $1 \cdot 11$ & $0.90,1 \cdot 36$ & $1 \cdot 12$ & $0 \cdot 91,1 \cdot 38$ & $0 \cdot 168$ \\
\hline Heavy drinkers & 1 & Ref. & $1 \cdot 38$ & $0 \cdot 91,2 \cdot 10$ & $1 \cdot 53$ & $1 \cdot 01,2 \cdot 33$ & $1 \cdot 79$ & $1 \cdot 18,2 \cdot 72$ & 0.007 \\
\hline \multicolumn{10}{|l|}{ Physical activity } \\
\hline \multicolumn{10}{|l|}{ No } \\
\hline Yes & 1 & Ref. & $1 \cdot 18$ & $0.99,1.40$ & $1 \cdot 16$ & $0.97,1.39$ & $1 \cdot 24$ & $1 \cdot 03,1 \cdot 49$ & 0.043 \\
\hline \multicolumn{10}{|l|}{ Dietary supplement use } \\
\hline Yes & 1 & Ref. & $1 \cdot 21$ & $1 \cdot 03,1 \cdot 43$ & $1 \cdot 22$ & $1 \cdot 03,1 \cdot 44$ & $1 \cdot 34$ & $1 \cdot 12,1 \cdot 59$ & 0.002 \\
\hline \multicolumn{10}{|l|}{ Healthy pattern } \\
\hline \multicolumn{9}{|l|}{ Non-smokers } & \\
\hline Ex-smokers & 1 & Ref. & 0.94 & $0.59,1.52$ & 0.69 & $0 \cdot 42,1 \cdot 16$ & 0.68 & $0 \cdot 40,1 \cdot 15$ & 0.079 \\
\hline Current smokers & 1 & Ref. & 0.75 & $0.55,1.03$ & 0.49 & $0.34,0.71$ & 0.54 & $0 \cdot 38,0 \cdot 78$ & $<0.001$ \\
\hline \multicolumn{10}{|l|}{ Alcohol consumption } \\
\hline \multicolumn{10}{|l|}{ Non-drinkers } \\
\hline Light drinkers & 1 & Ref. & 0.77 & $0.63,0.94$ & 0.88 & $0 \cdot 72,1 \cdot 07$ & 0.91 & $0 \cdot 75,1 \cdot 11$ & 0.708 \\
\hline Heavy drinkers & 1 & Ref. & 0.75 & $0.53,1.06$ & 0.57 & $0 \cdot 39,0.84$ & $0 \cdot 81$ & $0 \cdot 57,1 \cdot 16$ & $0 \cdot 138$ \\
\hline \multicolumn{10}{|l|}{ Physical activity } \\
\hline No & & & & & & & & & \\
\hline Yes & 1 & Ref. & $1 \cdot 42$ & $1 \cdot 20,1 \cdot 69$ & $1 \cdot 71$ & $1 \cdot 44,2 \cdot 03$ & $2 \cdot 03$ & $1 \cdot 70,2 \cdot 41$ & $<0.001$ \\
\hline \multicolumn{10}{|l|}{ Dietary supplement use } \\
\hline No & & & & & & & & & \\
\hline Yes & 1 & Ref. & $1 \cdot 38$ & $1 \cdot 17,1 \cdot 62$ & $1 \cdot 43$ & $1 \cdot 22,1 \cdot 68$ & $1 \cdot 81$ & $1 \cdot 54,2 \cdot 13$ & $<0.001$ \\
\hline \multicolumn{10}{|l|}{ Traditional pattern } \\
\hline \multicolumn{10}{|l|}{ Smoking } \\
\hline \multicolumn{10}{|l|}{ Non-smokers } \\
\hline Ex-smokers & 1 & Ref. & $1 \cdot 16$ & $0.70,1.93$ & 0.82 & $0 \cdot 48,1 \cdot 41$ & 0.98 & $0.58,1.64$ & 0.609 \\
\hline Current smokers & 1 & Ref. & 0.76 & $0.54,1.07$ & 0.70 & $0.50,0.99$ & 0.52 & $0 \cdot 36,0 \cdot 75$ & 0.001 \\
\hline \multicolumn{10}{|l|}{ Alcohol consumption } \\
\hline Non-drinkers & & & & & & & & & \\
\hline Light drinkers & 1 & Ref. & $1 \cdot 12$ & $0.91,1.37$ & $1 \cdot 26$ & $1 \cdot 03,1 \cdot 53$ & $1 \cdot 37$ & $1 \cdot 12,1 \cdot 67$ & 0.001 \\
\hline Heavy drinkers & 1 & Ref. & 0.66 & $0.46,0.95$ & 0.91 & $0 \cdot 64,1 \cdot 28$ & $0 \cdot 70$ & $0 \cdot 48,1 \cdot 01$ & $0 \cdot 186$ \\
\hline Physical activity & & & & & & & & & \\
\hline No & & & & & & & & & \\
\hline Yes & 1 & Ref. & 0.94 & $0 \cdot 80,1 \cdot 12$ & 0.91 & $0.77,1.08$ & $0 \cdot 84$ & $0.71,0.99$ & 0.038 \\
\hline Dietary supplement use & & & & & & & & & \\
\hline $\begin{array}{l}\text { NO } \\
\text { Yes }\end{array}$ & 1 & Ref. & $1 \cdot 26$ & $1 \cdot 07,1 \cdot 47$ & $1 \cdot 34$ & $1 \cdot 14,1 \cdot 57$ & $1 \cdot 16$ & $0 \cdot 98,1 \cdot 36$ & 0.057 \\
\hline
\end{tabular}

Ref., reference category.

${ }^{*}$ Adjusted for age.

tNon-drinkers $(0 \mathrm{~g} / \mathrm{d})$; light drinkers $(<12 \mathrm{~g} / \mathrm{d})$; and heavy drinkers $(\geq 12 \mathrm{~g} / \mathrm{d})$.

higher adherence to the Mediterranean (healthy) dietary pattern were less likely to increase their alcohol consumption $(\mathrm{OR}=0 \cdot 66,95 \% \mathrm{CI} 0 \cdot 46,0 \cdot 95)^{(16)}$. In contrast, the Multiethnic Cohort Study in Hawaii found that the fat and meat dietary pattern was positively associated with BMI, smoking and alcohol consumption ( $\geq 1$ drink/week; OR $=1 \cdot 40,95 \%$ CI $1 \cdot 37,1 \cdot 43)^{(14)}$.

For physical activity and dietary supplement use, our study found both of these factors to be positively associated with all dietary patterns in both genders, with the exception of physical activity, which was inversely associated with the traditional dietary pattern in women. The
EPIC-Potsdam study found that physical activity level was positively associated with the 'fruit and vegetable' dietary pattern in men $(\beta=0 \cdot 182)$ and was negatively associated with the 'bread and sausage' pattern in women $(\beta=0 \cdot 099)^{(10)}$. The Multiethnic Cohort Study found that physical activity ( $\geq 3$ times/week) was positively associated with the vegetable $(\mathrm{OR}=1 \cdot 73,95 \% \mathrm{CI} 1 \cdot 69,1 \cdot 77)$ and fruit and milk patterns (OR $=1 \cdot 44,95 \%$ CI $1 \cdot 40$, $1.47)$, but not with the fat and meat pattern $(\mathrm{OR}=0.98$, $95 \%$ CI $0 \cdot 96,1 \cdot 01)^{(14)}$. In addition, participants with the healthy pattern have been reported to have higher physical activity levels and to be more likely to take dietary 
supplements daily ${ }^{(27)}$. Likewise, in our study, physical activity was more strongly associated with the healthy dietary pattern than other dietary patterns in men $(\mathrm{OR}=$ $2 \cdot 16,95 \% \mathrm{CI} 1 \cdot 86,2 \cdot 53)$ and women $(\mathrm{OR}=2 \cdot 03,95 \% \mathrm{CI}$ $1 \cdot 70,2 \cdot 41$ ). Among 64252 women in the French E3N-EPIC cohort, supplement use was positively associated with the fruit/vegetable pattern and inversely associated with the processed meat/starchy foods and alcohol/meat products patterns $(P \text { for trend for all associations }<0 \cdot 001)^{(33)}$. In the German Nutrition Survey, comprising 7124 men and women, significant differences in food consumption between regular vitamin and mineral supplement users and non-users were observed, indicating a tendency towards a healthier diet among regular users of supplements ${ }^{(19)}$. In a study by McNaughton et l $^{(11)}$, dietary supplement use was positively associated with the fruit/vegetable pattern and inversely associated with a Western dietary pattern, and dietary supplement users tended to have a healthier lifestyle and diet than non-users.

Most dietary pattern studies have been conducted in North America or Europe, and only a few studies have been conducted in Asia and Korea. Thus, the results of the present study are important as they reflect dietary habits common in Asian countries. It is important to note that our study participants were volunteers recruited at one cancer screening centre; therefore, the results may not be generalizable to other Korean populations. However, the relatively high socio-economic status of our study population may contribute to more accurate responses regarding risk factors and may have helped to reduce errors related to internal validity. Yet the present study has a limitation. The FFQ focused on eating habits rather than actual food intake amounts. The FFQ did not ask about portion size, and therefore information on nutrient intake was not available. In addition, the use of an FFQ containing only sixteen food groups may restrict the number of food categories used to characterize the usual dietary intake. It is not clear to what degree this FFQ represents habitual food intake.

In summary, the present study found three different dietary patterns in a middle-aged Korean population and indicates that these dietary patterns are strongly associated with health behaviours. The healthy dietary patterns were mainly associated with other healthy lifestyle behaviour factors, including not smoking, low alcohol consumption, participating in physical activity and dietary supplement use. The possible confounding effect of other risk behaviours should be appropriately considered when conducting nutritional epidemiological studies of the association between dietary patterns and disease outcomes.

\section{Acknowledgements}

The present study was supported by grants from the National Cancer Center, Korea (Grant numbers 0610552 and 0910222). There are no conflicts of interest. A.S. and
J.K. designed the study; E.R.C. and S.-Y.L. conducted the statistical analysis; and E.R.C. wrote the manuscript. All authors critically reviewed and approved the manuscript.

\section{References}

1. Zarraga IG \& Schwarz ER (2006) Impact of dietary patterns and interventions on cardiovascular health. Circulation 114, 961-973.

2. Hu FB (2002) Dietary pattern analysis: a new direction in nutritional epidemiology. Curr Opin Lipidol 13, 3-9.

3. Newby PK \& Tucker KL (2004) Empirically derived eating patterns using factor or cluster analysis: a review. Nutr Rev 62, 177-203.

4. Cai H, Zheng W, Xiang YB et al. (2007) Dietary patterns and their correlates among middle-aged and elderly Chinese men: a report from the Shanghai Men's Health Study. BrJ Nutr 98, 1006-1013.

5. Heidemann C, Schulze MB, Franco OH et al. (2008) Dietary patterns and risk of mortality from cardiovascular disease, cancer, and all causes in a prospective cohort of women. Circulation 118, 230-237.

6. Osler M, Heitmann BL, Gerdes LU et al. (2001) Dietary patterns and mortality in Danish men and women: a prospective observational study. Br J Nutr 85, 219-225.

7. van Dam RM, Rimm EB, Willett WC et al. (2002) Dietary patterns and risk for type 2 diabetes mellitus in US men. Ann Intern Med 36, 201-209.

8. Terry P, Hu FB, Hansen H et al. (2001) Prospective study of major dietary patterns and colorectal cancer risk in women. Am J Epidemiol 154, 1143-1149.

9. Terry P, Suzuki R, Hu FB et al. (2001) A prospective study of major dietary patterns and the risk of breast cancer. Cancer Epidemiol Biomarkers Prev 10, 1281-1285.

10. Bamia C, Orfanos P, Ferrari P et al. (2005) Dietary patterns among older Europeans: the EPIC-Elderly study. Br J Nutr 94, 100-113.

11. McNaughton SA, Mishra GD, Stephen AM et al. (2007) Dietary patterns throughout adult life are associated with body mass index, waist circumference, blood pressure, and red cell folate. J Nutr 137, 99-105.

12. Pryer JA, Cook A \& Shetty P (2001) Identification of groups who report similar patterns of diet among a representative national sample of British adults aged 65 years of age or more. Public Health Nutr 4, 787-795.

13. Schulze MB, Hoffmann K, Kroke A et al. (2001) Dietary patterns and their association with food and nutrient intake in the European Prospective Investigation into Cancer and Nutrition (EPIC)-Potsdam study. Br J Nutr 85, 363-373.

14. Park SY, Murphy SP, Wilkens LR et al. (2005) Dietary patterns using the Food Guide Pyramid groups are associated with sociodemographic and lifestyle factors: the Multiethnic Cohort Study. J Nutr 135, 843-849.

15. Kim MK, Sasaki S, Sasazuki S et al. (2004) Prospective study of three major dietary patterns and risk of gastric cancer in Japan. Int J Cancer 110, 435-442.

16. Sanchez-Villegas A, Toledo E, Bes-Rastrollo M et al. (2009) Association between dietary and beverage consumption patterns in the SUN (Seguimiento Universidad de Navarra) cohort study. Public Health Nutr 12, 351-358.

17. Imamura F, Lichtenstein AH, Dallal GE et al. (2009) Confounding by dietary patterns of the inverse association between alcohol consumption and type 2 diabetes risk. $\mathrm{Am}$ J Epidemiol 170, 37-45.

18. Beitz R, Mensink GB, Hintzpeter B et al. (2004) Do users of dietary supplements differ from nonusers in their food consumption? Eur J Epidemiol 19, 335-341. 
19. Kerver JM, Yang EJ, Bianchi L et al. (2003) Dietary patterns associated with risk factors for cardiovascular disease in healthy US adults. Am J Clin Nutr 78, 1103-1110.

20. Shin KO, Oh SY \& Park HS (2007) Empirically derived major dietary patterns and their associations with overweight in Korean preschool children. Br J Nutr 98, 416-421.

21. Shin A, Lim SY, Sung J et al. (2009) Dietary intake, eating habits, and metabolic syndrome in Korean men. J Am Diet Assoc 109, 633-640.

22. Yang EJ, Kerver JM \& Song WO (2005) Dietary patterns of Korean Americans described by factor analysis. J Am Coll Nutr 24, 115-121.

23. Slattery ML, Boucher KM, Caan BJ et al. (1998) Eating patterns and risk of colon cancer. Am J Epidemiol 148, $4-16$.

24. Fung T, Hu FB, Fuchs C et al. (2003) Major dietary patterns and the risk of colorectal cancer in women. Arch Intern Med 163, 309-314.

25. Newby PK, Muller D, Hallfrisch J et al. (2003) Dietary patterns and changes in body mass index and waist circumference in adults. Am J Clin Nutr 77, 1417-1425.

26. Chen H, Ward MH, Graubard BI et al. (2002) Dietary patterns and adenocarcinoma of the esophagus and distal stomach. Am J Clin Nutr 75, 137-144.
27. van Dam RM, Grievink L, Ocke MC et al. (2003) Patterns of food consumption and risk factors for cardiovascular disease in the general Dutch population. Am J Clin Nutr 77, 1156-1163.

28. Engeset D, Alsaker E, Ciampi A et al. (2005) Dietary patterns and lifestyle factors in the Norwegian EPIC cohort: the Norwegian Women and Cancer (NOWAC) study. Eur J Clin Nutr 59, 675-684.

29. Knudsen VK, Rasmussen LB, Haraldsdottir J et al. (2002) Use of dietary supplements in Denmark is associated with health and former smoking. Public Health Nutr 5, 463-468.

30. Zondervan KT, Ocke MC, Smit HA et al. (1996) Do dietary and supplementary intakes of antioxidants differ with smoking status? Int J Epidemiol 25, 70-79.

31. Perrin AE, Dallongeville J, Ducimetiere P et al. (2005) Interactions between traditional regional determinants and socio-economic status on dietary patterns in a sample of French men. Br J Nutr 93, 109-114.

32. McClernon FJ, Westman EC, Rose JE et al. (2007) The effects of foods, beverages, and other factors on cigarette palatability. Nicotine Tob Res 9, 505-510.

33. Touvier M, Niravong M, Volatier JL et al. (2009) Dietary patterns associated with vitamin/mineral supplement use and smoking among women of the E3N-EPIC cohort. Eur J Clin Nutr 63, 39-47.

\section{Appendix}

\section{Food list for the sixteen food groups in the FFQ of the present study}

\begin{tabular}{ll}
\hline Food group & Food list \\
\hline Fast foods & Pizza, chicken, processed food \\
Animal fat-rich foods & High-fat red meat (beef, pork), processed food (ham, sausage), butter \\
Fried foods & Deep-fried food, stir-fried food \\
Grilled meat and seafood & Grilled ribs, barbecue, grilled squid \\
Sweet foods & Bread, cookies, chocolate, honey, candy, ice cream \\
Cholesterol-rich foods & Egg yolks, seafood (eel, shrimp, squid), organ meats (from fish or other animals) \\
Caffeinated drinks & Coffee, black tea, cocoa, coke \\
Green-yellow vegetables & Carrots, spinach, sesame leaf, lettuce, courgette, dropwort \\
Healthy protein foods & Lean meat, fish, tofu, legumes, egg whites \\
Seaweeds & Laver, brown seaweed, tangle \\
Bonefish & Anchovies, dried whitebait \\
Fruit & Apples, tangerines, grapes, watermelon, strawberries, peaches, pears, fruit juice \\
Dairy products & Yoghurt, cheese, milk \\
Salted vegetables and seafood & Kimchi, salted fermented seafood, food boiled in soya with spices \\
Cereals & Rice, bread, noodles, potatoes, sweet potatoes \\
Light-coloured vegetables & Bean sprouts, cucumbers, radishes, onions, bellflower \\
\hline
\end{tabular}

